\title{
IDENTITIES OF GROUP ALGEBRAS
}

\author{
PAUL MILNES ${ }^{1}$
}

\begin{abstract}
In this note the converse to the following well-known proposition about locally compact topological groups $G$ is proved: if $G$ is discrete, then the group $C^{*}$-algebra $C^{*}(G)$ has an identity.
\end{abstract}

Let $G$ be a locally compact topological group and let $L: f \rightarrow L_{f}$ denote the left regular representation of $L^{1}(G)$ on $L^{2}(G) ; L_{f} h$ $=f^{*} h, \forall h \in L^{2}(G)$. If $G$ is compact, it is a classical result that $L_{f}$ is a compact operator $\forall f \in L^{1}(G)$. If, as well, $G$ is not discrete, then $L^{2}(G)$ is infinite dimensional and, for each $f \in L^{1}(G)$, there is a sequence in $\left\{L_{f} h / h \in L^{2}(G),\|h\|_{2}=1\right\}$ converging to zero in $L^{2}(G)$. We now prove this last statement for arbitrary nondiscrete locally compact topological groups.

Proposition. Suppose $G$ is a nondiscrete locally compact topological group. Then, if $f \in L^{1}(G)$, there is a sequence in $\left\{L_{f} h / h \in L^{2}(G),\|h\|_{2}=1\right\}$ converging to zero in $L^{2}(G)$.

Proof. It suffices to prove the result for $f \in C_{00}(G)$, a norm-dense subset of $L^{1}(G)$. Suppose $f \in C_{00}(G),\|f\|_{\infty}=a$ and the support of $f$ is $K$, a compactum. Let $\left\{V_{n}\right\}$ be a decreasing sequence of compact symmetric neighbourhoods of the identity of $G$ such that $\mu\left(V_{n}\right) \rightarrow 0$, where $\mu$ is left Haar measure on $G$, and let $h_{n}=\chi_{V_{n}} /\left(\mu\left(V_{n}\right)\right)^{1 / 2}$. Then $L_{f} h_{n}(t)=f^{*} h_{n}(t)=\int f(s) h_{n}\left(s^{-1} t\right) d \mu(s)=0 \quad \forall t \in K V_{n}$ and, $\forall t \in G$, $\left|L_{f} h_{n}(t)\right| \leqq a\left(\mu\left(V_{n}\right)\right)^{1 / 2}$. Hence, $\left\|L_{f} h_{n}\right\|_{2}^{2} \leqq a^{2} \mu\left(V_{n}\right) \mu\left(K V_{n}\right) \rightarrow 0$ as $n \rightarrow \infty$. Q.E.D.

Let $C_{r}^{*}(G)$ denote the $C^{*}$-algebra obtained by completing $L^{1}(G)$ in the norm $f \rightarrow\left\|L_{f}\right\| . C_{r}^{*}(G)$ can be identified with the $C^{*}$-subalgebra of $\mathscr{L}\left(L^{2}(G)\right)$ generated by the operators $\left\{L_{f} / f \in L^{1}(G)\right\}$ (see $[1, \mathrm{p}$. 187 ], for example).

Corollary 1. Suppose $G$ is a nondiscrete locally compact topological group. Then $C_{r}^{*}(G)$ does not have an identity.

Proof. Suppose $C_{r}^{*}(G)$ does have an identity, 1. Let $f \in L^{1}(G)$ be such that $\left\|1-L_{f}\right\| \leqq 1 / 4$ and let $h \in L^{2}(G),\|h\|_{2}=1$ be such

Received by the editors October 26, 1970.

AMS 1970 subject classifications. Primary 22D25.

$K e y$ words and phrases. Locally compact topological group, $C^{*}$-algebra.

1 The author is indebted to Professor L. T. Gardner, his supervisor at the University of Toronto, where this research was done, for some helpful discussions.

Copyright () 1971. American Mathematical Society 
that $\left\|L_{f} h\right\|_{2} \leqq 1 / 4$. Then $\left\|\left(1-L_{f}\right) h\right\|_{2}=\left\|h-L_{f} h\right\|_{2} \geqq\|h\|_{2}-\left\|L_{f} h\right\|_{2} \geqq 3 / 4$, which is a contradiction.

The group $C^{*}$-algebra $C^{*}(G)$ of $G$ is obtained by completing $L^{1}(G)$ in the norm $f \rightarrow \sup \left\|\pi_{f}\right\|$, where $\pi$ ranges over all *-representations of $L^{1}(G)$ as operators on a Hilbert space.

Corollary 2. Suppose $G$ is a nondiscrete locally compact topological group. Then $C^{*}(G)$ does not have an identity.

Proof. Since $C_{r}^{*}(G)$ is a homomorphic image of $C^{*}(G)[1,(1.15)$, p. 187] and $C_{r}^{*}(G)$ has no identity, $C^{*}(G)$ has no identity.

It is well known that, if $G$ is discrete, $L^{1}(G)$ has an identity and hence so do $C_{r}^{*}(G)$ and $C^{*}(G)$.

\section{REFERENCES}

1. P. Eymard, L'algèbre de Fourier d'un groupe localement compact, Bull. Soc. Math. France 92 (1964), 181-236. MR 37 \#4208.

University of Western Ontario, London, Ontario, Canada 